\title{
LOCAL ONE-SIDED MAXIMAL FUNCTION ON FRACTIONAL SOBOLEV SPACES
}

\author{
ABHISHEK GHOSH AND KALACHAND SHUIN
}

Abstract. In this article we study the boundedness of local one sided maximal operators on weighted fractional Sobolev Spaces. As a consequence we obtain a Lebesgue differentiation theorem for functions in fractional Sobolev spaces.

Mathematics subject classification (2010): Primary 42B25, Secondary 46E35.

Keywords and phrases: Local maximal operator, $A_{p}$ weights, fractional Sobolev space.

\section{REFERENCES}

[1] L. Forzani, F. J. Martín-Reyes, S. Ombrosi, Weighted inequalities for the two-dimensional one-sided Hardy-Littlewood maximal function, Trans. Amer. Math. Soc. 363 (2011), no. 4, 16991719. 42B25 (37A40 47A35).

[2] Piotr Hajeasz, Jani Onninen, On boundedness of maximal functions in Sobolev spaces, Ann. Acad. Sci. Fenn. Math. 29 (2004), no. 1, 167-176. 42B20 (42B25 46E35).

[3] Juha Kinnunen, The Hardy-Littlewood maximal function of a Sobolev function, Israel J. Math. 100 (1997), 117-124, 30C65.

[4] Juha Kinnunen, Eero Saksman, Regularity of the fractional maximal function, Bull. London Math. Soc. 35 (2003), no. 4, 529-535. 42B35 (46E35 47G10).

[5] Juha Kinnunen, Peter Lindqvist, The derivative of the maximal function, J. Reine Angew. Math. 503 (1998), 161-167. 42B25.

[6] Hannes Luiro, On the regularity of the Hardy-Littlewood maximal operator on subdomains of $\mathbb{R}^{n}$, Proc. Edinb. Math. Soc. (2) 53 (2010), no. 1, 211-237. 42B25 (47H09).

[7] Hannes Luiro, Continuity of the maximal operator in Sobolev spaces, Proc. Amer. Math. Soc. 135 (2007), no. 1, 243-251. 42B25 (46E35 47B38).

[8] Hannes Luiro, AntTi V. VhKangas, Local maximal operators on fractional Sobolev spaces, J. Math. Soc. Japan 68 (2016), no. 3, 1357-1368. 42B25 (46E35).

[9] Hannes Luiro, Antti V. Vhrangas, Beyond local maximal operators, Potential Anal. 46 (2017), no. 2, 201-226. 42B25 (46E35 47H99).

[10] F. J. MARTÍN-REYES, New proofs of weighted inequalities for the one-sided Hardy-Littlewood maximal functions, Proc. Amer. Math. Soc. 117 (1993), no. 3, 691-698. 42B25.

[11] ONDŘEJ KURKA, On the variation of the Hardy-Littlewood maximal function, Ann. Acad. Sci. Fenn. Math. 40 (2015), no. 1, 109-133. 42B25 (42B30 46E35).

[12] B. MucKenHoupt, Weighted norm inequalities for the Hardy maximal function, Trans. Amer. Math. Soc. 165 (1972), 207-226.

[13] E. SAWYER, Weighted inequalities for the one-sided Hardy-Littlewood maximal functions, Trans. Amer. Math. Soc. 297 (1986), no. 4, 367-374.

[14] TANAKA Hitoshi, A remark on the derivative of the one-dimensional Hardy-Littlewood maximal function, Bull. Austral. Math. Soc. 65 (2002), no. 2, 253-258, 42B25.

[15] B. O. Turesson, Nonlinear potential theory and weighted Sobolev spaces, Lecture Notes in Mathematics, 1736, Springer-Verlag, Berlin, 2000. xiv+173 pp. 31C45 (35J70 46E35). 